\title{
Review: capillary refill time, abnormal skin turgor, and abnormal respiratory pattern help to detect dehydration in children
}

Steiner MJ, DeWalt DA, Byerley JS. Is this child dehydrated? JAMA 2004;291:2746-54.

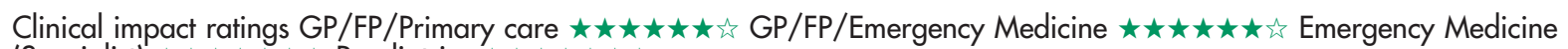

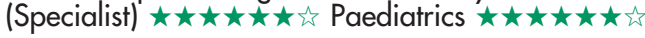

In children, what is the accuracy of signs, symptoms, and laboratory tests for detecting dehydration?

\section{METHODS}

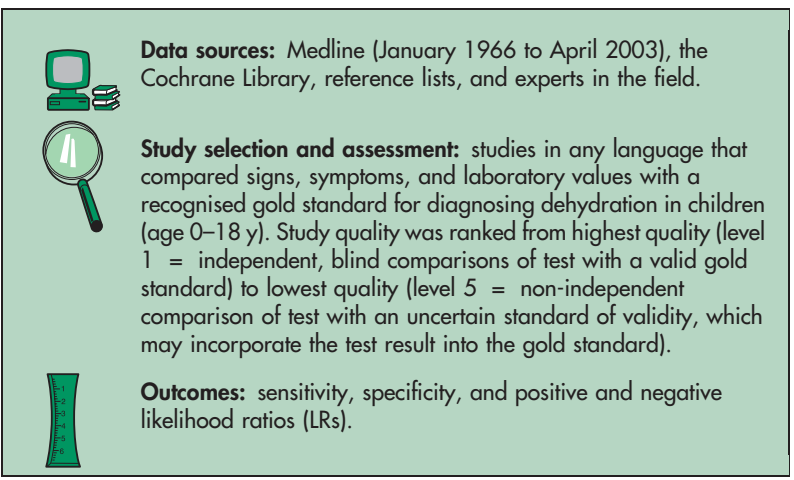

\section{MAIN RESULTS}

13 studies $(n=1246)$ met the selection criteria (4 intermediate quality and 9 low quality). Clinically useful signs for detecting 5\% dehydration were capillary refill time, abnormal skin turgor, and abnormal respiratory pattern (table). Dry mucous membranes, sunken eyes, and poor overall appearance are moderately useful in detecting $5 \%$ dehydration (table). 3 studies evaluated combinations of signs. In 1 study $(n=100)$, the combination of abnormal skin turgor, sunken eyes, dry mucous membranes, and a sunken fontanelle increased the likelihood of $10 \%$ dehydration (LR 3.7, $95 \%$ CI 1.6 to 8.1). Another study ( $\mathrm{n}=97$ ) showed that classification of "severe" on an assessment scale detected $\geqslant 5 \%$ dehydration in children who required intravenous fluids (LR 3.4, CI 1.5 to 7.7). In the third study $(\mathrm{n}=225)$, the combination of $\geqslant 3$ of 10 signs were useful for detecting 5\% dehydration (sensitivity $87 \%$, specificity $82 \%)$. In a pooled analysis of 3 studies $(\mathrm{n}=398)$, history taking (eg, parental report of low urine output) was not accurate for detecting the likelihood of 5\% dehydration (LR 1.3, CI 0.9 to 1.9). 6 studies evaluated laboratory tests; 5 studies $(\mathrm{n}=465)$ assessed blood urea nitrogen (BUN) or serum creatinine ratio. BUN cut points of 2.85, 6.42, and $9.63 \mathrm{mmol} / \mathrm{l}$ were moderately useful (LR range 1.4 to 2.9 ). Acidosis was not a useful test for detecting dehydration in 2 studies $(\mathrm{n}=221,+\mathrm{LR}<2.0)$. Another study $(\mathrm{n}=168)$ found that an absolute serum bicarbonate [SB] concentration $<15 \mathrm{mmol} / \mathrm{l}$ was not helpful for diagnosing $5 \%$ dehydration (LR for low SB 1.5, CI 1.2 to

For correspondence: Dr D A DeWalt, University of North Carolina School of Medicine, Chapel Hill, North Carolina, USA. dewaltd@med.unc.edu

Source of funding: Robert Wood Johnson Clinical Scholars Program.
1.9), while an absolute SB concentration of $<17 \mathrm{mmol} / \mathrm{l}$ was moderately helpful in another study $(\mathrm{n}=97)$ (LR 3.5, CI 2.1 to 5.8 ).

\section{CONCLUSIONS}

Capillary refill time, abnormal skin turgor, and abnormal respiratory pattern are the most useful signs for detecting dehydration. Combinations of signs perform better than individual signs. History taking and laboratory tests show modest utility.

\section{Commentary}

$T$ he review by Steiner et al focused on the clinical assessment of dehydration caused by acute gastroenteritis in children 1 month to 5 years of age. The well done review was limited primarily by the lack of high quality studies. The best 3 signs for assessing dehydration were prolonged capillary refill time, abnormal skin turgor, and abnormal respiratory pattern; parent reported symptoms and laboratory tests were usually not helpful in the initial assessment of dehydration.

The challenge to interpreting this literature is that study results are likely confounded by the rates of mild, moderate, and severe dehydration in their population. Likelihood ratios for some signs of dehydration varied by the degree of dehydration (rates of moderate to severe dehydration ranged from 6-48\%). In addition, acidosis can be secondary to either diarrhoea or dehydration; therefore the relative amounts of diarrhoea and vomiting leading to dehydration within study populations might explain why abnormal respirations were an important sign in some studies but not in others.

Although an accurate initial assessment of dehydration is undoubtedly important, it is not the only factor affecting physicians' treatment decisions. Unlike previous studies, the review concluded that ill appearance was not found to be a useful sign of dehydration. ${ }^{1}$ However, a lethargic infant is more likely to require further diagnostic evaluation and is less likely to be successfully orally hydrated.

Nevertheless, this evidence-based review does reflect the literature by supporting the use of signs of dehydration with proven diagnostic utility These might be organised conceptually as major criteria (abnormal capillary refill, abnormal respirations, or abnormal skin turgor) and minor criteria (some combination of ill appearance, dry mucous membranes, absent tears, and/or sunken eyes). To formulate a successful treatment plan, this assessment could then be combined with prognostic host factors (eg, lethargy and stage of illness) and family-related factors (eg, exhaustion and access to transportation).

Thomas M Ball, MD, MPH University of Arizona Tucson, Arizona, USA

1 Gorelick MH, Shaw KN, Murphy KO Validity and reliability of clinical sign in the diagnosis of dehydration in children. Pediatrics 1997:99:E6.

2 Duggan $C$, Refat $M$, Hashem $M$, et al. How valid are clinical signs of dehydration in infants? J Pediatr Gastroenterol Nutr 1996;22:56-61.

Test characteristics of examination signs to detect $5 \%$ dehydration in children (age range 2 weeks to 15 years)*

\begin{tabular}{llllll}
\hline Tests & Number of studies $(\mathbf{n})$ & Sensitivity (95\% CI) & Specificity (CI) & +LR & - LR \\
\hline Prolonged capillary refill & $4(478)$ & $60 \%(29$ to 91$)$ & $85 \%(72$ to 98$)$ & 4.0 & 0.47 \\
Abnormal skin turgor & $5(602)$ & $58 \%(40$ to 75$)$ & $76 \%(59$ to 93$)$ & 2.42 & 0.55 \\
Abnormal respiratory pattern & $4(581)$ & $43 \%(31$ to 55$)$ & $79 \%(72$ to 86$)$ & 2.05 & 0.72 \\
Dry mucous membranes & $4(533)$ & $86 \%(80$ to 92$)$ & $44 \%(13$ to 74$)$ & 1.54 & 0.32 \\
Sunken eyes & $4(533)$ & $75 \%(62$ to 88$)$ & $52 \%(22$ to 81$)$ & 1.56 & 0.48 \\
Poor overall appearance & $3(398)$ & $80 \%$ (57 to 104) & $45 \%(-10$ to 102) & 1.45 & 0.44 \\
\hline
\end{tabular}

*Diagnostic terms defined in glossary. LRs were calculated from data in article. 